QUASICONFORMAL GEOMETRY AND DYNAMICS

BANACH CENTER PUBLICATIONS, VOLUME 48

INSTITUTE OF MATHEMATICS

POLISH ACADEMY OF SCIENCES

WARSZAWA 1999

\title{
WHAT IS A DISK?
}

\author{
KARI HAG \\ Norwegian University of Science and Technology \\ N-7491 Trondheim, Norway \\ E-mail:kari@math.ntnu.no
}

Introduction. This paper should be considered as a companion report to F.W. Gehring's survey lectures "Characterizations of quasidisks" given at this Summer School [7]. Notation, definitions and background results are given in that paper. In particular, $D$ is a simply connected proper subdomain of $\mathbf{R}^{2}$ unless otherwise stated and $D^{*}$ denotes the exterior of $D$ in $\overline{\mathbf{R}}^{2}$.

Many of the characterizations of quasidisks have been motivated by looking at properties of euclidean disks. It is therefore natural to go back and ask if any of the original properties in fact characterize euclidean disks. We follow the procedure in Gehring's lectures and look at four different categories of properties:

1. Geometric properties,

2. Conformal invariants,

3. Injectivity criteria,

4. Extension properties.

As we shall see, the answers are not equally easy to obtain and not always positive. There are, in fact, still many interesting open questions.

\section{Geometric properties}

Reflection property

Characterization 1.1. D is a half plane if and only if there exists a euclidean isometry $f$ of $\overline{\mathbf{R}}^{2}$ which maps $D$ onto its exterior $D^{*}$ and is the identity on $\partial D$.

1991 Mathematics Subject Classification: Primary 46C20; Secondary 32G81.

A preliminary version of this paper has appeared in Bull. Soc. Sci. Lettres Łódź 47 Sér. Rech. Déform. 24 (1998), 67-72.

The paper is in final form and no version of it will be published elsewhere. 
Proof (sufficiency). Fix $z_{1} \in D$ and let $\zeta \in \partial D, z_{1}, \zeta \neq \infty$. Then

$$
\left|f\left(z_{1}\right)-\zeta\right|=\left|f\left(z_{1}\right)-f(\zeta)\right|=\left|z_{1}-\zeta\right| .
$$

Thus $\partial D \subset L \cup\{\infty\}$ where $L$ is the perpendicular bisector of the segment $\left[z_{1}, f\left(z_{1}\right)\right]$. On the other hand let $z \in L$ and consider the broken line $\gamma=\left[z_{1}, z\right] \cup\left[z, f\left(z_{1}\right)\right]$. Then $\gamma$ must meet $\partial D$ and also $\gamma \cap \partial D \subset \gamma \cap L$ which implies that $z \in \partial D$.

Reversed triangle inequality property. If $D$ is a disk or half plane, then

$$
\min _{j=1,2} \operatorname{dia}\left(\gamma_{j}\right) \leq\left|z_{1}-z_{2}\right|
$$

for any two points $z_{1}, z_{2} \in \partial D$ where $\gamma_{1}, \gamma_{2}$ are the components of $\partial D \backslash\left\{z_{1}, z_{2}\right\}$. In particular, $D$ satisfies Ahlfors's well known two point inequality [3] with constant 1 . But this inequality also holds if $D$ is a Reuleaux triangle, [6] and [15], and hence cannot be used to characterize a disk or half plane. However for the reversed triangle inequality [7] we have the following characterization.

Characterization 1.2. A Jordan domain $D \subset \mathbf{R}^{2}$ is a disk or half plane if and only if

$$
\left|z_{1}-z_{2}\right|\left|z_{3}-z_{4}\right|+\left|z_{2}-z_{3}\right|\left|z_{4}-z_{1}\right| \leq\left|z_{1}-z_{3}\right|\left|z_{2}-z_{4}\right|
$$

for each ordered quadruple of points $z_{1}, z_{2}, z_{3}, z_{4} \in \partial D \backslash\{\infty\}$.

Proof. For the sufficiency, choose three points on $\partial D \backslash\{\infty\}$ and let $C$ be the line or circle through these points. To complete the proof we shall show that each $z \in \partial D \backslash\{\infty\}$ lies on $C$.

Fix a point $z \in \partial D \backslash\{\infty\}$ different from the three points given above and label these four points $z_{1}, z_{2}, z_{3}, z_{4}$ so that they constitute an ordered quadruple in $\partial D$ with $z_{2}=z$. Next choose a Möbius transformation $f$ such that

$$
f\left(z_{1}\right)=0, \quad f\left(z_{3}\right)=1, \quad f\left(z_{4}\right)=\infty .
$$

Then $f(C)$ is the real axis $\mathbf{R}$ and it suffices to show that

$$
f\left(z_{2}\right) \in(0,1) \subset \mathbf{R} .
$$

By our hypothesis, the reversed triangle inequality yields

$$
\begin{aligned}
1 & \geq\left|\left(z_{1}, z_{4}, z_{2}, z_{3}\right)\right|+\left|\left(z_{2}, z_{1}, z_{3}, z_{4}\right)\right|=\left|\left(0, \infty, f\left(z_{2}\right), 1\right)\right|+\left|\left(f\left(z_{2}\right), 0,1, \infty\right)\right| \\
& =\left|f\left(z_{2}\right)\right|+\left|1-f\left(z_{2}\right)\right| \geq 1 .
\end{aligned}
$$

This means that we have equality throughout and hence that $f\left(z_{2}\right) \in(0,1)$.

To show that the condition is necessary, we reverse the last part of the above argument.

REMARK. The task of proving that the condition with equality is a necessary one is given as an exercise on p. 82 in [1].

Local connectivity property. Langmeyer has established the characterization given below in her thesis [15]. Our proof is a modification of hers.

Characterization 1.3. $D$ is a disk or half plane if and only if it is 1-linearly locally connected, i.e., for each $z_{0} \in \mathbf{R}^{2}$ and each $r>0$ 
a. points in $D \cap \bar{B}\left(z_{0}, r\right)$ can be joined by an arc in the same set,

b. points in $D \backslash B\left(z_{0}, r\right)$ can be joined by an arc in the same set.

Proof (sufficiency). The hypotheses imply that $D$ is a quasidisk and hence a Jordan domain [8]. In addition we may assume that $D$ is bounded since the property of being 1-linearly locally connected is preserved under Möbius transformations [24].

Property a. implies that $D$ is convex. To prove this fix $z_{1}, z_{2} \in D$ and let $\alpha$ be an arc joining these points in $D$. It follows that every point in a bounded component of $\mathbf{R}^{2} \backslash\left\{\left[z_{1}, z_{2}\right] \cup \alpha\right\}$ is in $D$. For consider a point $\zeta$ in such a domain, i.e., bounded by a segment $\left[z_{1}^{\prime},, z_{2}^{\prime}\right]$ of $\left[z_{1}, z_{2}\right]$ and a subarc $\alpha^{\prime}$ of $\alpha$. Let $U$ be a disk with $z_{1}^{\prime}, z_{2}^{\prime} \in \partial U$ and with radius so large that $\zeta \notin \bar{U}$. Since $D$ has property a. there is an arc $\alpha^{\prime \prime}$ in $D \cap \bar{U}$ joining $z_{1}^{\prime}$ and $z_{2}^{\prime}$. Then $\zeta$ is in a component of $\mathbf{R}^{2} \backslash\left\{\alpha^{\prime} \cup \alpha^{\prime \prime}\right\}$ which lies in $D$ and $\zeta \in D$ since $D$ is simply connected. Thus $\left[z_{1}, z_{2}\right] \subset \bar{D}$ and this implies that $\bar{D}$ is convex. Hence $D=\operatorname{Int}(\bar{D})$ is also convex.

Next we claim that

$$
\min \left\{\left|z_{1}-z_{0}\right|,\left|z_{3}-z_{0}\right|\right\} \leq \max \left\{\left|z_{2}-z_{0}\right|,\left|z_{4}-z_{0}\right|\right\}
$$

for all $z_{0} \in \mathbf{R}^{2}$ and all ordered quadruples of points $z_{1}, z_{2}, z_{3}, z_{4} \in \partial D$. For suppose there exist five points for which (1) does not hold, and let $r$ be a number such that

$$
\max \left\{\left|z_{2}-z_{0}\right|,\left|z_{4}-z_{0}\right|\right\}<r<\min \left\{\left|z_{1}-z_{0}\right|,\left|z_{3}-z_{0}\right|\right\} .
$$

Since $D$ is convex, $\beta=\left[z_{2}, z_{4}\right]$ is a crosscut in $D$. Hence any arc in $D$ joining points close to $z_{1}$ and $z_{3}$ must intersect $\beta \subset B\left(z_{0}, r\right)$ and cannot be in $D \backslash B\left(z_{0}, r\right)$, in contradiction to property b.

To finish up the proof choose $z_{1}, z_{3} \in \partial D$ such that

$$
\left|z_{1}-z_{3}\right|=\operatorname{dia}(D)=d
$$

and let $z_{0}$ denote the midpoint of $\left[z_{1}, z_{3}\right]$. The perpendicular bisector of $\left[z_{1}, z_{3}\right]$ intersects $\partial D$ in two points $z_{2}$ and $z_{4}$ lying in different components of $\partial D \backslash\left\{z_{1}, z_{3}\right\}$. Let $z_{0}^{\prime}$ be the midpoint of $\left[z_{2}, z_{4}\right]$. Then

$$
\frac{d}{2} \leq\left|z_{1}-z_{0}^{\prime}\right|=\left|z_{3}-z_{0}^{\prime}\right| \leq\left|z_{2}-z_{0}^{\prime}\right|=\left|z_{4}-z_{0}^{\prime}\right| \leq \frac{d}{2}
$$

where the middle inequality follows from (1) above. We must have equality throughout, and $z_{2}, z_{4}$ are diametrically opposite points on the circle through $z_{0}$ with radius $d / 2$. By (*) at most one of the four arcs $\widetilde{z}_{1} z_{2}, \widetilde{z}_{2} z_{3}, \widetilde{z}_{3} \widetilde{z}_{4}, \widetilde{z}_{4} \bar{z}_{1}$ of $\partial D$ can have a point closer to $z_{0}$ than $d / 2$. Hence we have two opposite arcs where this is not the case. They must both be on the circle $\left|z-z_{0}\right|=d / 2$ since otherwise $\operatorname{dia}(D)>d$. For any two diametrically opposite points on these arcs we can repeat the above argument with the perpendicular bisector. Therefore all the points on the two remaining arcs are also on the circle.

Decomposition property

Characterization 1.4. $D$ is a disk or half plane if and only if for each $z_{1}, z_{2} \in D$ there exists a disk $D^{\prime}$ with

$$
z_{1}, z_{2} \in D^{\prime} \subset D
$$


Proof (sufficiency). The hypotheses imply that $D$ is a quasidisk and hence a Jordan domain [11]. Next we may assume that $D$ is bounded. (If not we apply a suitable Möbius transformation.) We choose $z_{j}, z_{j}^{\prime} \in D$ such that

$$
\left|z_{j}-z_{j}^{\prime}\right| \rightarrow \operatorname{dia}(D)<\infty .
$$

By hypothesis we get $w_{j}, r_{j}$ such that

$$
z_{j}, z_{j}^{\prime} \subset B\left(w_{j}, r_{j}\right) \subset D
$$

and by passing to subsequences we may assume

$$
z_{j} \rightarrow z_{0}, z_{j}^{\prime} \rightarrow z_{0}^{\prime}, w_{j} \rightarrow w_{0}, r_{j} \rightarrow r_{0}
$$

Then $B\left(w_{0}, r_{0}\right) \subset \bar{D}$ and since $D$ has no inner boundary $B\left(w_{0}, r_{0}\right) \subset D$. In particular $2 r_{0} \leq \operatorname{dia}(D)$ but by construction $2 r_{0} \geq \operatorname{dia}(D)$. We conclude that $B\left(w_{0}, r_{0}\right)=D$.

2. Conformal invariants. There are three different cases when a Jordan domain $D$ together with some distinguished interior and/or boundary points has one conformal invariant. See [2] or [7].

(i) Two interior points $z_{1}, z_{2}$. The conformal invariant is the hyperbolic distance $h_{D}\left(z_{1}, z_{2}\right)$ between $z_{1}$ and $z_{2}$.

(ii) One interior point $z_{0}$ and the boundary points $z_{1}, z_{2}$. The conformal invariant is $\omega\left(z_{0}, \alpha ; D\right)$, the harmonic measure of the positively oriented boundary arc $\alpha=z_{1} \overparen{z_{2}}$ evaluated at $z_{0}$.

(iii) Four positively oriented boundary points $z_{1}, z_{2}, z_{3}, z_{4}$. The conformal invariant is the modulus of the quadrilateral $Q=D\left(z_{1}, z_{2}, z_{3} . z_{4}\right)$.

We will describe how each of these invariants can be used to characterize disks.

Hyperbolic bound property

Conjecture 2.1. $D$ is a disk if and only if

$$
h_{D}\left(z_{1}, z_{2}\right) \leq j_{D}\left(z_{1}, z_{2}\right)
$$

for $z_{1}, z_{2} \in D$ where $j_{D}$ is the metric given by

$$
j_{D}\left(z_{1}, z_{2}\right)=\log \left(\frac{\left|z_{1}-z_{2}\right|}{\operatorname{dist}\left(z_{1}, \partial D\right)}+1\right)\left(\frac{\left|z_{1}-z_{2}\right|}{\operatorname{dist}\left(z_{2}, \partial D\right)}+1\right) .
$$

ThEOREM 2.2 [10]. The above conjecture is true if in addition, for one pair of points $w_{1}, w_{2} \in \partial D$ with $\left|w_{1}-w_{2}\right|=\operatorname{dia}(D)$ there exist disks $D_{1}, D_{2} \subset D$ with $w_{1} \in \partial D_{1}$ and $w_{2} \in \partial D_{2}$.

\section{Harmonic symmetry property}

Characterizhtion 2.3. A Jordan domain $D$ is a disk or a half plane if and only if there exist points $z_{0} \in D$ and $z_{0}^{*} \in D^{*}$ such that if $\alpha$ and $\beta$ are adjacent open arcs in $\partial D$ with

$$
\omega\left(z_{0}, \alpha ; D\right)=\omega\left(z_{0}, \beta ; D\right),
$$

then

$$
\omega\left(z_{0}^{*}, \alpha ; D^{*}\right)=\omega\left(z_{0}^{*}, \beta ; D^{*}\right) .
$$


Proof (sufficiency). We consider conformal mappings $f: B \rightarrow D, g: B^{*} \rightarrow D^{*}$ such that $f(0)=z_{0}, g(\infty)=z_{0}^{*}$. Then $f, g$ have homeomorphic extensions to $\bar{B}, \bar{B}^{*}$ and by a preliminary rotation we may assume that $f(1)=g(1)$. Then

$$
h=g^{-1} \circ f: \partial B \rightarrow \partial B
$$

is a sense preserving homeomorphism such that $h(1)=1$. We want to show that $h(z)=z$.

For this let $\alpha, \beta$ be upper and lower halves of $\partial B$ labeled so that $i \in \alpha$. By conformal invariance of harmonic measure

$$
\omega\left(z_{0}, f(\alpha), D\right)=\omega(0, \alpha, B)=1 / 2=\omega(0, \beta, B)=\omega\left(z_{0}, f(\beta), D\right)
$$

and by hypothesis

$$
\begin{aligned}
\omega(0, \bar{h}(\alpha), B) & =\omega\left(\infty, h(\alpha), B^{*}\right)=\omega\left(z_{0}^{*}, g(h(\alpha)), D^{*}\right) \\
& =\omega\left(z_{0}^{*}, f(\alpha), D^{*}\right)=\omega\left(z_{0}^{*}, f(\beta), D^{*}\right) \\
& =\omega\left(z_{0}^{*}, g(h(\beta)), D^{*}\right)=\omega\left(\infty, h(\beta), B^{*}\right)=\omega(0, \bar{h}(\beta), B) .
\end{aligned}
$$

In particular, this implies $h(-1)=-1$ and, since $h$ is sense preserving, we have $h(\alpha)=\alpha$.

Next let $\alpha, \beta$ be left and right halves of the upper half of $\partial D$ labeled so that $e^{i \pi / 4} \in \alpha$. Then as above

$$
\omega\left(z_{0}, f(\alpha), D\right)=\omega(0, \alpha, B)=1 / 4=\omega(0, \beta, B)=\omega\left(z_{0}, f(\beta), D\right)
$$

and

$$
\omega(0, \bar{h}(\alpha), B)=\omega\left(z_{0}^{*}, f(\alpha), D^{*}\right)=\omega\left(z_{0}^{*}, f(\beta), D^{*}\right)=\omega(0, \bar{h}(\beta), B) .
$$

In particular we get that $h(i)=i$, and $h(\alpha)=\alpha$. Proceeding this way we see that

$$
h\left(e^{2 \pi i t}\right)=e^{2 \pi i t}
$$

for all $t \in[0,1)$ of the form $t=2^{-n} p, p \in \mathbf{Z}$, and by continuity $h(z)=z$. This implies that the conformal mappings $f$ and $g$ together define a homeomorphism $\varphi$ of $\overline{\mathbf{R}}^{2}$ which is conformal in $B \cup B^{*}$ and hence in $\overline{\mathbf{R}}^{2}$. In particular $\varphi$ is a Möbius transformation and $D=\varphi(B)$ is a disk or half plane.

Conjugate quadrilateral property. We recall that a quadrilateral $Q=D\left(z_{1}, z_{2}, z_{3}, z_{4}\right)$ consists of a Jordan domain $D \subset \overline{\mathbf{R}}^{2}$ together with four positively oriented points $z_{1}, z_{2}, z_{3}, z_{4} \in \partial D$. Moreover $Q^{*}=D^{*}\left(z_{4}, z_{3}, z_{2}, z_{1}\right)$ is called the conjugate quadrilateral. Any quadrilateral $Q$ can be mapped conformally onto a rectangle $R=R(0, m, m+i, i)$, and we call $m$ the $\operatorname{modulus}$ of $Q, \bmod (Q)=m$.

Characterization 2.4. A Jordan domain $D$ is a disk or half plane if and only if for each pair of conjugate quadrilaterals $Q$ and $Q^{*}$ in $D$ and $D^{*}$,

$$
\bmod \left(Q^{*}\right) \leq 1
$$

whenever $\bmod (Q)=1$.

Proof (sufficiency). Since

$$
\bmod \left(D^{*}\left(z_{4}, z_{3}, z_{2}, z_{1}\right)\right)=\frac{1}{\bmod \left(D^{*}\left(z_{3}, z_{2}, z_{1}, z_{4}\right)\right)},
$$

the hypotheses actually imply that $\bmod \left(Q^{*}\right)=1$ whenever $\bmod (Q)=1$. 
Let $f$ and $f^{*}$ be homeomorphic maps of $\bar{D}$ and $\bar{D}^{*}$ to the closed upper and lower half plane, respectively, which are conformal in $D$ and $D^{*}$ and normalized so that $w(0)=0$, $w(1)=1, w(\infty)=\infty$ where $w=f^{*} \circ f^{-1}$. If $z_{j}=f^{-1}\left(x_{j}\right)$ where $x_{1}<x_{2}<x_{3}$ and $x_{4}=\infty$, then

while

$$
\bmod \left(D\left(z_{1}, z_{2}, z_{3}, z_{4}\right)\right)=1 \text { if and only if } x_{2}=\frac{x_{1}+x_{3}}{2}
$$

and thus

$$
\bmod \left(D^{*}\left(z_{4}, z_{3}, z_{2}, z_{1}\right)\right)=1 \quad \text { if and only if } \quad w\left(x_{2}\right)=\frac{w\left(x_{1}\right)+w\left(x_{3}\right)}{2}
$$

$$
w\left(\frac{x_{1}+x_{3}}{2}\right)=\frac{w\left(x_{1}\right)+w\left(x_{3}\right)}{2} .
$$

Since $w(1)=1, w(0)=0$ we see that $w(x)=x$ for all $x \in \mathbf{R}$ of the form $x=2^{-n} p$, $p \in \mathbf{Z}$, and by continuity $w(x)=x$ for all $x \in \mathbf{R}$. This implies that the maps $f^{-1}$ and $f^{*-1}$ coincide on $\mathbf{R}$ and together define a conformal mapping in $\overline{\mathbf{R}}^{2}$, i.e., a Möbius transformation. It follows that $D$ is a disk or half plane.

Extremal distance. The modulus of a quadrilateral can also be defined using families of curves. We shall now consider the more general situation where we have defined for any family $\Gamma$ of curves in $\overline{\mathbf{R}}^{2}$ a modulus, $\bmod (\Gamma)$, which is conformally invariant.

Characterization 2.5 [25]. D is a disk or half plane if and only if

$$
\bmod (\Gamma) \leq 2 \bmod \left(\Gamma_{D}\right)
$$

whenever $E, F$ are continua in $D$ and $\Gamma_{D}, \Gamma$ denote the families of all curves which join $E$ and $F$ in $D$ and in $\overline{\mathbf{R}}^{2}$, respectively.

\section{Injectivity criteria}

Injectivity of analytic functions. Two different criteria for global injectivity of functions $f$ analytic in a domain $D$ are considered in [7]. The first involves the Schwarzian derivative $S_{f}$ and the second the pre-Schwarzian derivative $T_{f}=f^{\prime \prime} / f^{\prime}$. For each domain $D$ we define two constants $\sigma(D)$ and $\tau(D)$ as follows:

$\sigma(D)$ is the supremum of the constants $a \geq 0$ such that $f$ is injective whenever $f$ is meromorphic and locally injective with $\left|S_{f}\right| \leq a \rho_{D}^{2}$ in $D$.

$\tau(D)$ is the supremum of the constants $b \geq 0$ such that $f$ is injective whenever $f$ is analytic and locally injective with $\left|T_{f}\right| \leq b \rho_{D}$ in $D$.

Here $\rho_{D}$ denotes the hyperbolic density with curvature -1 in $D$. Then

$$
0 \leq \sigma(D) \leq 1 / 2 \text { and } 0 \leq \tau(D) \leq 1 / 2
$$

for all $D ;[16]$ and [23]. Moreover both constants are positive if and only if $D$ is a quasidisk. See [3], [8] and [4]

Characterization 3.1 [16], [19]. D is a disk or half plane if and only if

$$
\sigma(D)=1 / 2 \text {. }
$$

The situation for the pre-Schwarzian derivative is somewhat different. 
THEOREM 3.2 [5]. If $D$ is a disk or half plane, then

$$
\tau(D)=1 / 2 \text {. }
$$

But the converse of this result is not true.

THEOREM 3.3 [23]. If $f$ is conformal with $\left|z T_{f}(z)\right| \leq 1 / 2$ in $B$, then $\tau(f(B))=1 / 2$.

The following is an immediate consequence of Theorem 3.3.

EXAmPLE 3.4. If $D=f(B)$ where $f(z)=e^{z / 2}$, then $\tau(D)=1 / 2$.

Injectivity of local quasi-isometries. We recall that $f$ is a local $L$-quasi-isometry in $D$ if each point in $D$ has a neighborhood such that

$$
\frac{1}{L}\left|z_{1}-z_{2}\right| \leq\left|f\left(z_{1}\right)-f\left(z_{2}\right)\right| \leq L\left|z_{1}-z_{2}\right|
$$

for points $z_{1}, z_{2}$ in this neighborhood. Furthermore we denote by $L(D)$ the supremum of the numbers $L$ such that $f$ is injective whenever $f$ is a local $L$-quasi-isometry in $D$.

$L(D)>1$ if and only if $D$ is a quasidisk ([9] and [18]). $L(D)$ is not known when $D$ is a disk or half plane, much less that it can be used to characterize such domains. We have, however, the following results.

THEOREM 3.5 [13]. If $D$ is a disk or half plane, then $L(D) \geq 2^{1 / 4}$.

LEMMA 3.6. If $D$ is convex, then $L(D) \leq 2^{1 / 2}$.

Proof. Let $z_{0} \in D$, choose $z_{1} \in \partial D$ so that $\left|z_{1}-z_{0}\right|=\operatorname{dist}\left(z_{0}, \partial D\right)$, and let $T$ be the corresponding support plane for $D$ at $z_{1}$. Without loss of generality we may assume that $z_{1}=0$ and $T=\{z: \Re(z)>0\}$. Then for each $L>2^{1 / 2}$,

$$
f(z)=\frac{|z|}{L} \exp \left(i L^{2} \arg (z)\right)
$$

is a local $L$-quasi-isometry in $D$ which is not injective.

\section{Extensions}

Comparable Dirichlet integral property

Characterization 4.1. Let $D$ be a Jordan domain in $\mathbf{R}^{2}$. Then $D$ is a disk or half plane if and only if

$$
\int_{D^{*}}\left|\operatorname{grad} u^{*}\right|^{2} d m=\int_{D}|\operatorname{grad} u|^{2} d m
$$

for each pair of functions $u$ and $u^{*}$ which are harmonic in $D$ and $D^{*}$, respectively, with continuous and equal boundary values.

ProOF (sufficiency). By the conjugate quadrilateral characterization we are done if we can prove that

$$
\bmod \left(D\left(z_{1}, z_{2}, z_{3}, z_{4}\right)\right)=\bmod \left(D^{*}\left(z_{4}, z_{3}, z_{2}, z_{1}\right)\right)
$$

for all positively oriented quadruples $z_{1}, z_{2}, z_{3}, z_{4} \in \partial D$.

For this choose $u$ harmonic in $D$ and continuous in $\bar{D}$ with boundary values 0 and 1 , respectively, on the Jordan arcs $z_{2} z_{3}$ and $z_{4} z_{1}$. Next let $f$ be the canonical mapping of 
$Q$ onto the rectangle $R=R(0, m, m+i, i)$ and set $v=u \circ f^{-1}$. Then

$$
\int_{0}^{m}|\operatorname{grad} v| d x \geq 1
$$

for $0<y<1$, and with equality if $v=x / m$. Thus

$$
1 \leq \int_{0}^{1}\left(\int_{0}^{m}|\operatorname{grad} v| d x\right) d y \leq\left(\int_{R}|\operatorname{grad} v|^{2} d m\right)^{1 / 2} \bmod (Q)^{1 / 2}
$$

and

$$
\frac{1}{\bmod (Q)}=\inf _{v} \int_{R}|\operatorname{grad} v|^{2} d m=\inf _{v} \int_{D}|\operatorname{grad} v|^{2}\left|f^{\prime}\right|^{2} d m=\inf _{u} \int_{D}|\operatorname{grad} u|^{2} d m .
$$

Similarly

Hence our assumption implies that

$$
\frac{1}{\bmod \left(Q^{*}\right)}=\inf _{u^{*}} \int_{D^{*}}\left|\operatorname{grad} u^{*}\right|^{2} d m .
$$

$$
\bmod \left(Q^{*}\right)=\bmod (Q),
$$

and the result follows as remarked above.

REMARK. Since

$$
\bmod \left(D\left(z_{2}, z_{3}, z_{4}, z_{1}\right)\right)=\frac{1}{\bmod \left(D\left(z_{1}, z_{2}, z_{3}, z_{4}\right)\right)}
$$

the result is implied by

$$
\int_{D^{*}}\left|\operatorname{grad} u^{*}\right|^{2} d m \leq \int_{D}|\operatorname{grad} u|^{2} d m
$$

Extension of functions with bounded Dirichlet integral

ChARACTERIZATION 4.2. D is a disk or half plane if and only if each locally integrable function $u$ with bounded Dirichlet integral in $D$ has an extension to a locally integrable function $v$ with bounded Dirichlet integral in $\mathbf{R}^{2}$ such that

$$
\int_{\mathbf{R}^{2}}|\operatorname{grad} v|^{2} d m \leq 2 \int_{D}|\operatorname{grad} u|^{2} d m .
$$

Proof (sufficiency). The hypotheses imply that $D$ is a quasidisk and hence a Jordan domain. See [12] or [14].

Now consider a pair of functions $u$ and $u^{*}$ harmonic in $D$ and $D^{*}$, respectively, with continuous and equal boundary values and suppose that

$$
\int_{D}|\operatorname{grad} u|^{2} d m<\infty
$$

By the assumption, $u$ has an extension to a function $v$ such that

$$
\int_{D^{*}}|\operatorname{grad} v|^{2} d m+\int_{D}|\operatorname{grad} u|^{2} d m \leq 2 \int_{D}|\operatorname{grad} u|^{2} d m
$$

whence

$$
\int_{D^{*}}|\operatorname{grad} v|^{2} d m \leq \int_{D}|\operatorname{grad} u|^{2} d m
$$


Next by the Dirichlet Principle,

$$
\int_{D^{*}}\left|\operatorname{grad} u^{*}\right|^{2} d m \leq \int_{D^{*}}|\operatorname{grad} v|^{2} d m .
$$

Thus

$$
\int_{D^{*}}\left|\operatorname{grad} u^{*}\right|^{2} d m \leq \int_{D}|\operatorname{grad} u|^{2} d m
$$

and the result follows from the Remark above.

\section{Extension of conformal mappings}

Characterization 4.3. $D$ is a disk or half plane if and only if every conformal self mapping of $D$ has a conformal extension to $\overline{\mathbf{R}}^{2}$.

Our proof depends on the following characterization for Jordan domains.

Lemma 4.4. $D$ is a Jordan domain if and only if every conformal self mapping of $D$ has a homeomorphic extension to $\bar{D}$.

Proof (sufficiency). By hypothesis, each conformal self mapping of $D$ has a homeomorphic extension to $\bar{D}$. We shall show that $D$ is a Jordan domain, or equivalently, by Theorem 2.6 of [21], that $\partial D$ is locally connected and has no cut points.

To prove that $\partial D$ is locally connected, we consider a conformal map $g: B \rightarrow D$. By Theorem 1.7 of [21] there exists $z_{0} \in \partial B$ such that

$$
\lim _{r \rightarrow 1} g\left(r z_{0}\right)=w_{0}
$$

Next let $z_{1} \in \partial B$ and set

$$
h(z)=\frac{z_{1}}{z_{0}} z .
$$

Then by our hypothesis,

$$
f=g \circ h \circ g^{-1}: D \rightarrow D
$$

has a homeomorphic extension $f^{*}: \bar{D} \rightarrow \bar{D}$. In particular,

$$
\lim _{r \rightarrow 1} g\left(r z_{1}\right)=\lim _{r \rightarrow 1} g \circ h\left(r z_{0}\right)=\lim _{r \rightarrow 1} f^{*} \circ g\left(r z_{0}\right)=f^{*}\left(w_{0}\right)=w_{1} .
$$

Since $z_{1}$ was an arbitrary point in $\partial B$, we conclude that $g$ has a radial limit at each point $z \in \partial B$. Then Proposition 2.23 of [21] implies that there exists a point $z_{0} \in \partial B$ at which $g$ has in fact a limit and, arguing as above, we see that $g$ has a limit at each point $z \in \partial B$. Thus $g$ has a continuous extension to $\bar{D}$ and $\partial D$ is locally connected by Theorem 2.1 of [21].

Finally by Theorem IV.11.1 of [20], there exists $w_{0} \in \partial D$ which is not a cut point of $\partial D$. But then $\partial D$ is cut point free. For let $w_{1}$ be any point in $\partial D \backslash\left\{w_{0}\right\}$ and choose $z_{0}, z_{1} \in \partial B$ such that $g\left(z_{0}\right)=w_{0}$ and $g\left(z_{1}\right)=w_{1}$. As before, $w_{1}=f^{*}\left(w_{0}\right)$,

$$
\partial D \backslash\left\{w_{1}\right\}=f^{*}(\partial D) \backslash\left\{f^{*}\left(w_{0}\right)\right\}=f^{*}\left(\partial D \backslash\left\{w_{0}\right\}\right)
$$

is connected and hence $w_{1}$ is not a cut point of $\partial D$.

Proof of Characterization 4.3. For the sufficiency it is enough to prove that $D$ is a disk when $D$ is bounded since $D$ is a Jordan domain by Lemma 4.4. 
Now pick any disk $U \subset D$ with two boundary points $z_{1}, z_{2} \in \partial D$ and choose $w_{1}, w_{2} \in$ $\partial D$ such that $\left|w_{1}-w_{2}\right|=\operatorname{dia}(D)$. Then because $D$ is a Jordan domain, there exists a conformal self mapping $f$ of $D$ such that $f^{*}\left(z_{j}\right)=w_{j}$ for $j=1,2$ where $f^{*}$ is the homeomorphic extension of $f$ of $\bar{D}$. By our hypothesis, $f^{*}$ is the restriction of a Möbius transformation to $D$ and $f(U)$ must be a disk in $D$ with diameter equal to dia $(D)$. It follows that $f(U)=D$.

To be in accordance with the characterization of quasidisks as quasiconformal extension domains [22] we can reformulate the above result as follows.

CHARACTERIZATION 4.5. $D$ is a disk or half plane if and only if each $K$-quasiconformal self mapping of $D$ has a K-quasiconformal extension to $\overline{\mathbf{R}}^{2}$.

\section{References}

[1] L. V. Ahlfors, Complex analysis, McGraw-Hill 1979.

[2] L. V. Ahlfors, Conformal invariants, McGraw-Hill 1973.

[3] L. V. Ahlfors, Quasiconformal reflections, Acta Math. 109 (1963), 291-301.

[4] K. Astala and F. W. GEHRING, Injectivity, the BMO norm and the universal Teichmüller space, J. d'Analyse Math. 46 (1986), 16-57.

[5] J. Becker and Ch. Pommerenke, Schlichtheitskriterien und Jordangebiete, J. reine angew. Math. 354 (1984), 74-94,

[6] M. Berger, Geometry I and II, Springer-Verlag 1980.

[7] F. W. GeHring, Characterizations of quasidisks, this volume.

[8] F. W. Gehring, Univalent functions and the Schwarzian derivative, Comm. Math. Helv. 52 (1977), 561-572.

[9] F. W. Gehring, Injectivity of local quasi-isometries, Comm. Math. Helv. 57 (1982), 202220 .

[10] F. W. GeHring and K. HAG, Hyperbolic geometry and disks, in: Proceedings of the Conference on Continued Fractions and Geometric Function Theory - Trondheim (to appear).

[11] F. W. Gehring and B. G. Osgood, Uniform domains and the quasihyperbolic metric, J. d'Analyse Math. 36 (1979), 50-74.

[12] V. M. Gol'dstein and S. K. Vodop'Janov, Prolongement des fonctions de classe $L_{1}^{p}$ et applications quasi conformes, C. R. Acad. Sc. Paris 290 (1980), 453-456.

[13] F. John, On quasi-isometric mappings II, Comm. Pure Appl. Math. 22 (1969), 265-278.

[14] P. W. Jones, Quasiconformal mappings and extendability of functions in Sobolev spaces, Acta Math. 147 (1981), 71-88.

[15] N. LAngmeyer, The quasihyperbolic metric, growth, and John domains, Univ. Michigan Thesis 1996.

[16] M. Lehtinen, On the inner radius of univalency for non-circular domains, Ann. Acad. Sci Fenn. 5 (1980), 45-47.

[17] O. Lehto and K. I. ViRTAnen, Quasiconformal mappings in the plane, Springer-Verlag 1973.

[18] O. Martio and J. Sarvas, Injectivity theorems in plane and space, Ann. Acad. Sci. Fenn. 4 (1978-79), 383-401. 
[19] Z. NehaRI, The Schwarzian derivative and schlicht functions, Bull. Amer. Math. Soc. 55 (1949), 545-551.

[20] M. H. A. Newman, The topology of plane point sets, Cambridge University Press 1954.

[21] Ch. Pommerenke, Boundary behaviour of conformal maps, Springer-Verlag 1992.

[22] S. Rickman, Extensions over quasiconformally equivalent curves, Ann Acad. Sci. Fenn. 10 (1985), 511-514.

[23] D. Stowe, Injectivity and the pre-Schwarzian derivative, Mich. Math. J. 45 (1998), 537546.

[24] M. WALKER, Linearly locally connected sets and quasiconformal mappings, Ann. Acad. Sci. Fenn. 11 (1986), 77-86.

[25] S. YANG, QED domains and NED sets in $\bar{R}^{n}$, Trans. Amer. Math. Soc. 334 (1992), $97-120$. 